\title{
Plasma and Fecal Metabolite Profiles in Autism Spectrum Disorder
}

\author{
Brittany D. Needham, Mark D. Adame, Gloria Serena, Destanie R. Rose, Gregory M. Preston, \\ Mary C. Conrad, A. Stewart Campbell, David H. Donabedian, Alessio Fasano, Paul Ashwood, \\ and Sarkis K. Mazmanian
}

\begin{abstract}
BACKGROUND: Autism spectrum disorder (ASD) is a neurodevelopmental condition with hallmark behavioral manifestations including impaired social communication and restricted repetitive behavior. In addition, many affected individuals display metabolic imbalances, immune dysregulation, gastrointestinal dysfunction, and altered gut microbiome compositions.

METHODS: We sought to better understand nonbehavioral features of ASD by determining molecular signatures in peripheral tissues through mass spectrometry methods (ultrahigh performance liquid chromatography-tandem mass spectrometry) with broad panels of identified metabolites. Herein, we compared the global metabolome of 231 plasma and 97 fecal samples from a large cohort of children with ASD and typically developing control children. RESULTS: Differences in amino acid, lipid, and xenobiotic metabolism distinguished ASD and typically developing samples. Our results implicated oxidative stress and mitochondrial dysfunction, hormone level elevations, lipid profile changes, and altered levels of phenolic microbial metabolites. We also revealed correlations between specific metabolite profiles and clinical behavior scores. Furthermore, a summary of metabolites modestly associated with gastrointestinal dysfunction in ASD is provided, and a pilot study of metabolites that can be transferred via fecal microbial transplant into mice is identified.

CONCLUSIONS: These findings support a connection between metabolism, gastrointestinal physiology, and complex behavioral traits and may advance discovery and development of molecular biomarkers for ASD.
\end{abstract}

https://doi.org/10.1016/j.biopsych.2020.09.025

Many diseases are associated with informative metabolic signatures, or biomarkers, that enable diagnoses, predict disease course, and guide treatment strategies. In contrast, autism spectrum disorder (ASD) is diagnosed based on observational evaluation of behavioral symptoms, including reduced social interaction and repetitive/stereotyped behaviors (1). The average age of ASD diagnosis is between 3 and 4 years old (2), at which time children can receive behavioral therapy, the gold standard treatment. Because earlier diagnosis improves efficacy of behavioral therapies $(3,4)$, molecular biomarkers represent an attractive approach for identifying atrisk populations and may aid development of personalized therapies. This prospect is increasingly important given the rising rate of ASD diagnoses, which currently stands at up to 1 in 59 children in the United States (2), with high variability of worldwide estimates (approximately 1\%-2\%) $(5,6)$ and no Food and Drug Administration-approved drugs for core behavioral symptoms.

Metabolic abnormalities have been reported in ASD (7), though most studies have measured a small subset of metabolites, and many outcomes have not been reproduced between cohorts. Mitochondrial dysfunction, which heavily influences systemic metabolism, is estimated to be higher in ASD individuals than control subjects (5\% vs. approximately $0.01 \%$ ) (8), and genes crucial for mitochondrial function are risk factors for ASD (9). The metabolic abnormalities associated with mitochondrial dysfunction in ASD affect cellular energy, oxidative stress, and neurotransmission in the gut and the brain (9-21). Other metabolic profiles in ASD implicate aromatic and phenolic metabolites, including derivatives of nicotinic acid, amino acid, and hippurate metabolism (22-32). Various amino acids are detected at differential levels across studies and across sample types, but consistent patterns are difficult to discern $(17,26,28,31,33-36)$.

Some discrepancies between studies are likely due to differences in sample number, tissue analyzed, and methodology. Other sources of variability include differences in environmental factors, such as diet and gut bacteria, which differ between ASD and typically developing (TD) populations and can influence each other (37-39). Diet is a major source of circulating metabolites, impacting the metabolome directly or indirectly through chemical transformation by the trillions of gut microbes, the microbiome, which has been proposed to modulate complex behaviors (40-42). Such proposed environmental modulators of ASD may integrate with genetic risks 
to impact behavioral end points through the actions of small molecules produced in tissues outside the brain.

Herein, we present a comprehensive comparison of an extensive panel of identified metabolites in human plasma and feces from a large cohort of matched children with ASD and TD children. We identified differential levels of metabolites ranging from hormones, amino acids, xenobiotics, and lipids, many of which correlate with clinical behavior scores. To our knowledge, this is the first study to concurrently evaluate paired intestinal and systemic metabolomes in a high-powered analysis with a large number of identified metabolites, allowing direct associations between metabolites previously highlighted in ASD samples and discovering new metabolites of interest. These findings support the emerging concept of evaluating nonbehavioral features in the diagnosis of ASD.

\section{METHODS AND MATERIALS}

Samples for this study, from children 3 to 12 years of age, were collected through the MIND Institute, University of California, Davis $(43,44)$. ASD diagnosis was confirmed by trained staff using the Autism Diagnostic Observation Schedule (ADOS) and the Autism Diagnostic Interview-Revised. Diagnosis made in subjects before 2013 was based on DSM-IV. TD participants were screened using the Social Communication Questionnaire and scored within the typical range $(<15)$. An additional evaluation to determine gastrointestinal (Gl) symptoms was completed by 97 participants who also provided stool samples. Gl status was determined using the Gl symptom scale, based on Rome III Diagnostic Questionnaire on Pediatric Functional Gastrointestinal Disorders (45) and described in detail elsewhere (44). See Supplemental Methods in Supplement 1 for further information.

\section{RESULTS}

\section{Plasma and Fecal Metabolomes Differ Between ASD and TD}

Plasma samples from 130 children with ASD and 101 TD children were analyzed along with fecal samples collected from a subset of these same children with ASD $(n=57)$ and TD children $(n=40)$ (Figure S1A-G in Supplement 1). Samples and metrics of behavioral and Gl scores were obtained from the MIND institute $(43,44)$. The ASD group was stratified into subsets of children with Gl symptoms (ASD+Gl) or without GI symptoms (ASD-GI), to explore potential effects of comorbid intestinal dysfunction in ASD (40 of 130 ASD samples were $A S D+G I)$. This stratification was based on symptoms associated with $A S D$, including diarrhea, constipation, and irritable bowel syndrome-like symptoms.

Samples were analyzed by the global metabolite panel (plasma and fecal samples) and the complex lipid panel (plasma samples) from Metabolon, Inc. (Morrisville, NC), which identified a total panel of 1611 plasma and 814 fecal metabolites (Tables S1-S6 in Supplement 2). Overall, we discovered that 194 metabolites were differentially abundant between the ASD and TD groups in plasma and 19 metabolites were differentially abundant in feces (Figure 1A, B; Tables S1 and S4 in Supplement 2). Using a quantitative assay for a targeted panel of metabolites, we observed a high correlation between relative abundance and precise concentration $\left(r^{2}=\right.$ approximately .97) for all tested metabolites except $N$-acetylserine $\left(r^{2}=.63\right)$ (Figure $\mathrm{S} 1 \mathrm{H}$ in Supplement 1). Overall, these data expand on previous evidence that the metabolomic profiles of ASD and TD populations display differences not only in the gut compartment but also in circulation, which may affect the levels of metabolites throughout the body, including the brain $(46,47)$.

We then used random forest machine learning analysis to determine if metabolite profiles can predict without bias whether the sample came from an ASD or a TD donor. Overall, the modest predictive accuracy of this machine learning approach was $69 \%$ for plasma and $67 \%$ for feces. To test whether focusing on the most discriminating metabolites would improve the prediction, we repeated the random forest analysis using the top 30 metabolites and found that the predictive accuracy for plasma improved slightly to $70 \%$ when using all ASD samples and to $74 \%$ when using only ASD - Gl samples. For fecal samples, the predictive accuracy improved to $75 \%$ using all ASD samples and to $67 \%$ using only ASD - Gl samples. The top 30 metabolites, calculated by measuring the mean decrease in accuracy of the machine learning algorithm, are useful to describe the strongest drivers of overall metabolic differences between ASD and TD populations. These most discriminatory metabolites were primarily from the lipid, amino acid, xenobiotic, and cofactor/vitamin super pathways (Figure 1E, F). Several of these metabolites have been previously linked to $A S D$, such as steroids, bile acids, acylcarnitines, and nicotinamide metabolites (48-51). Further, multiple molecules known to be produced or manipulated by the gut microbiota also featured prominently, including 4ethylphenyl sulfate (4-EPS), which is elevated in an ASD mouse model, and indolelactate, a microbe-derived tryptophan metabolite (Figure 1E) $(52,53)$. The two most discriminatory molecules in plasma (Figure 1G, H) and feces (Figure 11, J) are depicted, which are detected in almost every sample except for 9-HOTre, which has a slightly lower percent fill in ASD compared with TD samples (84\% vs. $91 \%)$. Metabolites correlating most strongly with these discriminatory metabolites are closely related on a structural and metabolic level (Figure 1K, L).

\section{Metabolite Levels Correlate With Clinical Behavioral Scores}

Using clinical metadata for children with ASD, we correlated the levels of individual metabolites to the verbal, social, and nonverbal scores of the following standard diagnostic measures, all conducted by trained health professionals: the Autism Diagnostic Interview-Revised (a parent questionnaire) and the cumulative ADOS severity score (SS) (Tables S1, S4 in Supplement 2) (1). To contextualize the biological relevance of different metabolomes between ASD and TD samples, individual metabolites were integrated into biochemical pathways for pathway enrichment analysis, revealing the degree of change within each. We identified changes mostly in lipid, xenobiotic, and nucleotide pathways associated with diverse physiological processes (Figure S2C in Supplement 1). We found that verbal and social scores primarily correlate with lipid metabolism pathways and that nonverbal scores have the 
A

\begin{tabular}{|c|c|c|c|}
\hline Compared to TD & 1 & $\downarrow$ & Total \\
\hline All ASD & 31 & 163 & 194 \\
\hline ASD-GI & 29 & 91 & 120 \\
\hline ASD+GI & 18 & 97 & 115 \\
\hline
\end{tabular}

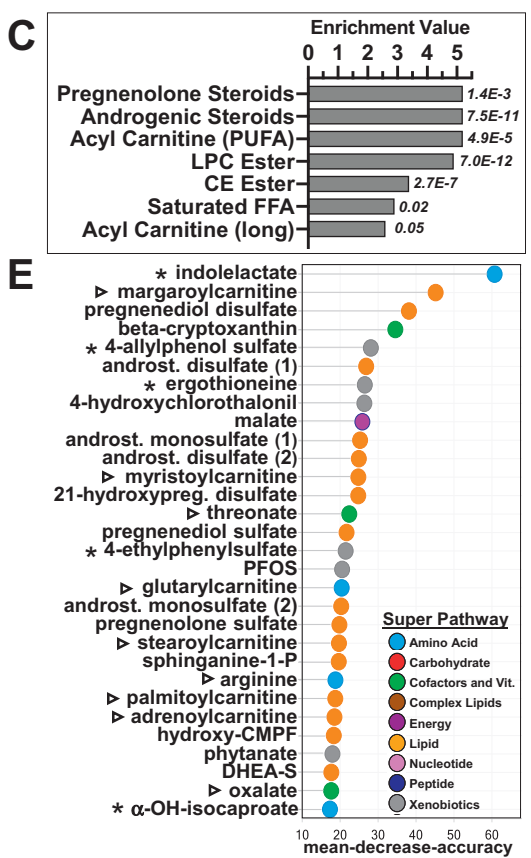

G

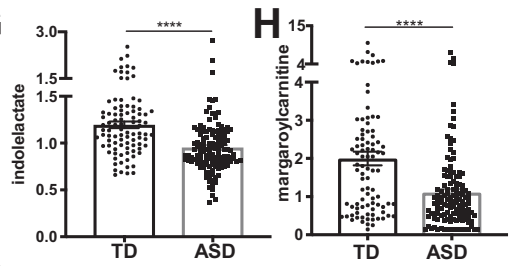

K

\begin{tabular}{|c|c|c|c|}
\hline & Correlated Metabolite & $\mathbf{r}$ & qval \\
\hline \multirow{8}{*}{ 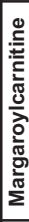 } & myristoylcarnitine (C14) & 0.88 & *** \\
\hline & stearoylcarnitine (C18) & 0.88 & *** \\
\hline & palmitoylcarnitine (C16) & 0.87 & $* * *$ \\
\hline & palmitoleoylcarnitine $(\mathrm{C} 16: 1)$ & 0.81 & $* * *$ \\
\hline & oleoylcarnitine (C18:1) & 0.79 & *** \\
\hline & cysteinylglycine & 0.78 & *** \\
\hline & $\operatorname{LPC}(17: 0)$ & 0.77 & *** \\
\hline & dihomo-linoleoylcarnitine (C20:2) & 0.77 & *** \\
\hline \multirow{8}{*}{ 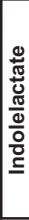 } & phenyllactate (PLA) & 0.51 & ** \\
\hline & 3-(4-hydroxyphenyl)lactate & 0.51 & ** \\
\hline & alpha-hydroxyisovalerate & 0.48 & ** \\
\hline & 2-hydroxy-3-methylvalerate & 0.46 & ** \\
\hline & $\mathrm{N}$-acetyltryptophan & 0.41 & ** \\
\hline & kynurenate & 0.40 & ** \\
\hline & imidazole lactate & 0.38 & ** \\
\hline & $\operatorname{LPC}(14: 0)$ & 0.38 & ** \\
\hline
\end{tabular}

B

Feces
\begin{tabular}{|c|c|c|c|}
\hline Compared to TD & \multicolumn{1}{|c|}{} & $\boldsymbol{1}$ & Total \\
\hline All ASD & 7 & 12 & 19 \\
\hline ASD-GI & 3 & 5 & 8 \\
\hline ASD+GI & 4 & 3 & 7 \\
\hline
\end{tabular}
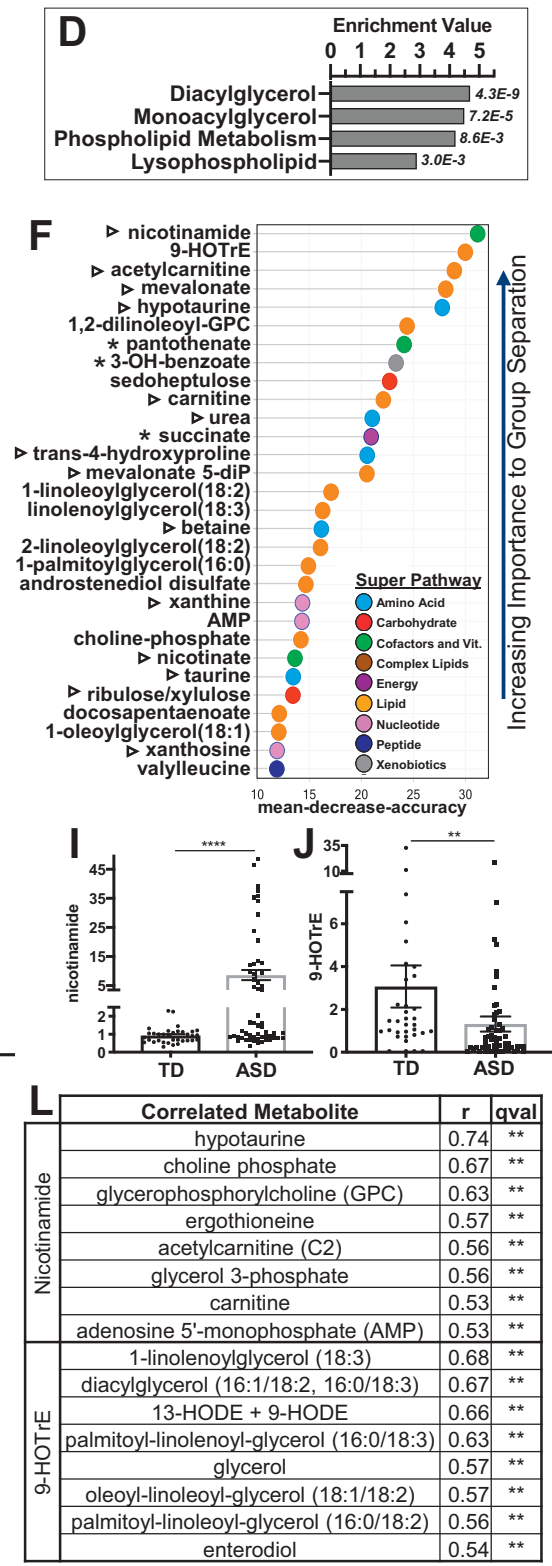

Figure 1. Plasma and fecal metabolomes differ between autism spectrum disorder (ASD) and typically developing (TD) groups. (A, B) The number of significantly elevated and decreased metabolites $(p$ $<.05, q<.1$ in ASD samples compared with the TD control group by analysis of variance contrasts in plasma and feces, respectively. Samples are stratified by all samples or samples without or with gastrointestinal (Gl) symptoms (-Gl, +Gl). (C, D) Pathway analysis results of human plasma and fecal comparisons (all samples), indicating which metabolomic pathways are the most altered between groups, with enrichment value plotted and $p$ value to the right of each bar. Metabolites within each pathway could be observed at either higher or lower levels, as this plot indicates only changes. (E, F) Top 30 most distinguishing metabolites between each group in plasma and feces by random forest analysis, with mean decrease accuracy along the $x$-axis, which is determined by randomly permuting a variable, running the observed values through the trees, and then reassessing the prediction accuracy. If a variable is important to the classification, the prediction accuracy will drop after such a permutation. Metabolites known to be produced by (asterisks) or influenced by (triangles) the gut microbiota are denoted. The super pathway to which each metabolite belongs is indicated by color of sphere and defined in the legend. (G, H) Scaled intensity values indicating relative levels of the most distinguishing molecules between ASD and TD (all samples) in plasma. Asterisks indicate significance $(p<.05, q<.1)$ in analysis of variance contrasts performed on total metabolomics dataset. Data are represented as mean \pm SEM. (I, J) Scaled intensity values indicating relative levels of the most distinguishing molecules between ASD and TD (all samples) in feces. Asterisks indicate significance $(p<.05, q<.1)$ in analysis of variance contrasts performed on total metabolomics dataset. Data are represented as mean \pm SEM. (K) Top correlated plasma metabolites that covary with margaroylcarnitine and indolelactate. (L) Top correlated fecal metabolites that covary with nicotinamide and 9-HOTrE. AMP, adenosine monophosphate; androst., androstane; CE, cholesterol ester; DHEA-s, dehydroepiandrosterone sulfate; FFA, free fatty acid; hydroxypreg., hydroxypregnenalone; LPC, lysophosphatidylcholine; PFOS, perfluorooctanesulfonic acid; PUFA, polyunsaturated fatty acid; qval, $q$ value. fewest correlations. Verbal scores show modest, negative correlations with levels of individual metabolites, including various plasma glycerolipids (i.e., diacylglycerols, glycerophosphorylcholine, monoacylglycerols), and both plasma and fecal levels of bile acids and sphingolipids. However, these correlations are not particularly striking on an individual level
(Figure S2D in Supplement 1; Tables S1, S4 in Supplement 2). Social scores positively correlate with total free fatty acid levels in plasma, most significantly with a C18 chain length (Figure S2E in Supplement 1; Table S1 in Supplement 2). After false discovery rate correction, no individual metabolites significantly correlated with nonverbal score. 


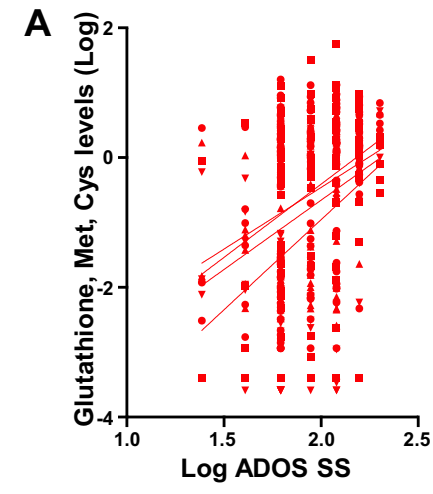

\begin{tabular}{|rr|c|c|c|}
\hline & Metabolite & Corr & pval & qval \\
\hline$*$ & Cys-gly, oxidized & 0.32 & $3 \mathrm{E}-4$ & 0.056 \\
\hline$*$ & cystine & 0.31 & $5 \mathrm{E}-4$ & 0.06 \\
\hline$*$ & Cysteinylgly disulfide & 0.30 & $7 \mathrm{E}-4$ & 0.08 \\
\hline- & Cysteine-GSSG & 0.29 & $8 \mathrm{E}-4$ & 0.08 \\
\hline
\end{tabular}

B
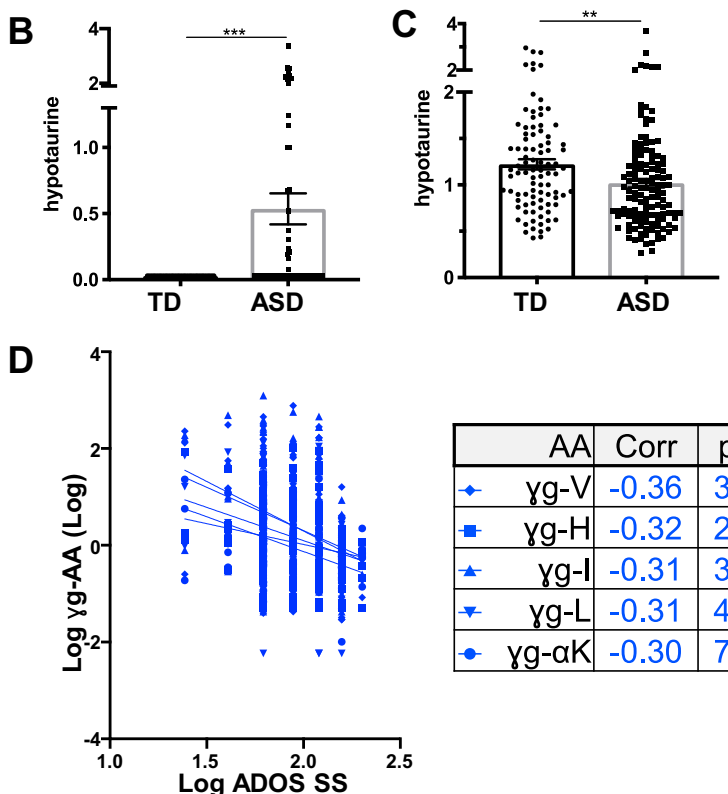

E

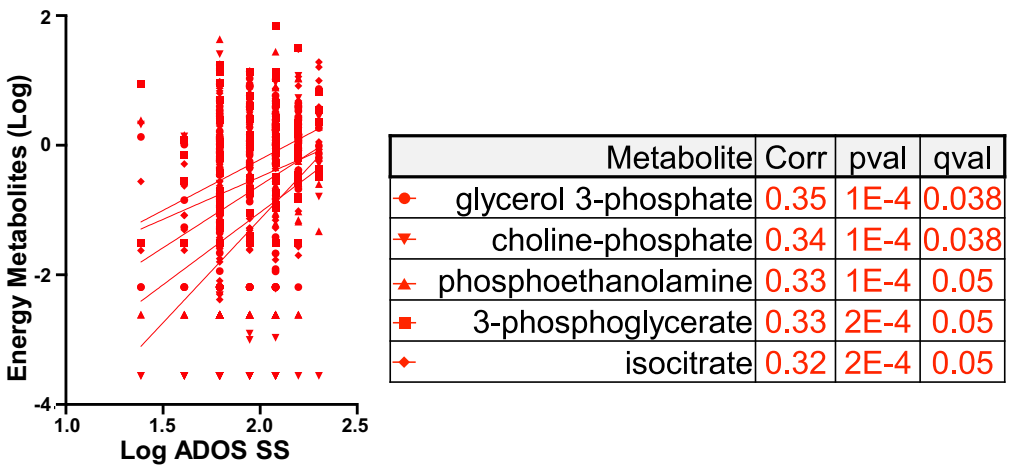

Positive correlation: $\mathrm{p}<0.05, \mathrm{q}<0.1$ Negative correlation: $\mathrm{p}<0.05, \mathrm{q}<0.1$
Figure 2. Metabolite levels correlate with Autism Diagnostic Observation Schedule (ADOS) severity score (SS). (A) Correlation of ADOS SS with metabolites from the cysteine, methionine, and glutathione pathways. Significant metabolites corresponding to the linear regression in the graph are listed along with Spearman coefficients and $p$ values. Refer to color legend at bottom. (B, C) Scaled intensity values indicating relative levels of hypotaurine in feces (B) and plasma (C) (all samples). Data are represented as mean \pm SEM. Asterisks indicate significance in analysis of variance contrasts performed on total metabolomics dataset with a false discovery rate cutoff of $q<.1\left(^{* *} p<.01\right.$, $\left.{ }^{* \star *} p<.001\right)$. (D) Correlations of gamma-glutamyl amino acids with ADOS SS, with Spearman coefficients and $p$ values to the right. Refer to color legend at bottom. (E) The top 5 most positively correlated plasma metabolites with ADOS SS, with Spearman coefficients and $p$ values to the right. Refer to color legend at bottom. AA, amino acid; Corr, correlation; Cys, cysteine; Cys-gly, cysteinylglycine; GSSG, glutathione disulfide; Met, methionine; pval, $p$ value; qval, $q$ value.
The ADOS SS correlated with diverse metabolite pathways, including amino acids and food/plant component pathways (Figure S2C in Supplement 1), which may be partly due to the diverse array of symptoms integrated into the ADOS SS score. On an individual metabolite level, we observed significant positive correlations and trends between the ADOS SS and metabolites in pathways of oxidative stress (cysteine, methionine, S-adenosyl-L-methionine, and glutathione pathways) (Figure 2A; Table S1 in Supplement 2). Some of these molecules were found in higher levels in the ASD fecal samples and at lower levels in the ASD plasma samples, such as hypotaurine and, to a lesser degree, taurine (Tables S1 and S4 in 
Supplement 2; Figure 2B, C; Figure S3A, B in Supplement 1), which might indicate altered fecal production, excretion, or differential uptake into the plasma potentially through varied intestinal permeability. Taurine plays many roles throughout the host and has previously been measured at altered levels in ASD, although with little consensus $(19,20,26-28,35,54-56)$. Hypotaurine and taurine deficiency has been shown to lead to defects in cell differentiation in the brain (56), and their dysregulation could alter neuronal signaling (57).

Dysregulated amino acid degradation, homeostasis, and import into the brain have been implicated as a cause of neuronal stress in ASD, and supporting metabolomic data have shown perturbations of various amino acid pathways, such as glutamate, methionine, glutathione, and gammaglutamyl metabolites $(12,19,26,28,31,35,51,58,59)$, some of which show associations with the ADOS SS (Table S1 in Supplement 2). Oxidative stress-related glutathione pathway precursors, gamma-glutamyl amino acids, can influence levels of neurotransmitters such as GABA (gamma-aminobutyric acid) $(57,60)$, which is widely thought to play a role in ASD. In a recent ASD study, an experimental treatment that led to increased gamma-glutamyl amino acids and other redox pathway metabolites correlated with improved behavior metrics in children (54). Here, we observed significant negative correlations and trends between many gamma-glutamyl amino acids and the ADOS SS (Figure 2D; Table S1 in Supplement 2). We also observed some perturbations in the urea cycle, which processes the amino group of amino acids for excretion in urine (Figure S3C, D in Supplement 1). Abnormalities in this pathway can be indicative of altered amino acid degradation observed in ASD and can lead to neurotoxic accumulation of nitrogen-containing compounds in the blood (61).

Additionally, the 5 plasma metabolites most positively correlated with ADOS SS all are involved in cellular energy pathways (Figure 2E; Table S1 in Supplement 2). Differences in energy markers could indicate a neurodevelopmental phenotype during periods when the high lipid and energy requirement in the brain is crucial (62-64). Overall, these correlations support the involvement of lipid, amino acid, and xenobiotic metabolism in the etiology of ASD, as previously described $(10,50,58)$, and reveal new candidates for ASD biomarkers that correlate with symptom severity.

\section{Altered Levels of Cellular Energy and Oxidative Stress Metabolites in ASD}

In line with the observed correlations between oxidative stress and cellular energy with the ADOS-SS, we further observed altered levels of related metabolites between ASD and TD samples. Metabolites that are markers of mitochondrial and oxidative stress can offer a snapshot into cellular metabolic states. These markers include acylcarnitines, which have been highlighted in various ASD studies and are established indicators of mitochondrial dysfunction $(13,14,16-20,26,27,35,48,55,65-69)$. Acylcarnitines are formed to allow transport of lipids across the mitochondrial membranes for beta-oxidation, and abnormal levels of these conjugated lipids accumulate to higher levels with a decrease in beta-oxidation. Interestingly, high levels of short acylcarnitines are found in rodent models where ASD-like behaviors are induced with the short-chain fatty acids valproic and propionic acids (70).

We found differential levels of acylcarnitines in ASD, creating a pattern of more abundant short-chain acylcarnitines and less abundant long-chain acylcarnitines in the ASD-GI samples compared with TD samples (Figure 3A). Acylcarnitines trend toward positive correlations with more severe social behavior, an effect driven by structures with shorter moieties (C2-C14) (Figure S2C in Supplement 1; Table S1 in Supplement 2). In fecal samples, acetylcarnitine (C2) and free carnitine were elevated in ASD (Figure 3B, C) and were highly discriminatory (Figure 1F). Other mitochondrial markers in both plasma and feces were also differentially abundant in ASD (Figure 3D) along with markers of phospholipid metabolism, which occurs largely in the mitochondria and was an enriched pathway in fecal comparisons (Figures 3D and 1D). Such defects in cellular metabolism support the theory that mitochondrial dysfunction may not only be comorbid with ASD but also may be a potential contributing factor, as suggested by numerous previous reports $(8,15,66,68,70)$, and alterations to levels of tricarboxylic acid cycle intermediates have been observed in human ASD prefrontal cortex samples (51). Together with the observed correlations between metabolites and the ADOS SS, these results corroborate and extend a growing body of research into altered mitochondrial metabolism and oxidative stress in ASD.

\section{Transfer of ASD Fecal Microbiota Into Mice Is Accompanied by Metabolic Signatures}

As microbial metabolites ranked highly in the random forest machine learning analysis, we tested if any of the observed metabolite differences in humans could be transferred to mice via fecal microbial transplant. We selected 4 male donor samples from each of the ASD and TD groups and colonized 2 or 3 male germ-free mice per donor for 3 weeks before collecting plasma and fecal samples for metabolite profiling and bacterial DNA sequencing, respectively (Figure S4A in Supplement 1). Global metabolomic analysis revealed that colonized mice modestly cluster by donor and group when differential metabolites are considered in principal component analysis (Figure S4B in Supplement 1). We selected the human donors based on 4-EPS levels (Figure S4C in Supplement 1), owing to the involvement of 4-EPS in an ASD mouse model (52) and dysregulation of similar phenolic compounds in human ASD $(24,26,30,31,71)$. 4-EPS is not produced by the host and is strictly a bacterial metabolite $(52,72)$. Surprisingly, we observed 4-EPS levels in a bimodal distribution in mouse samples (Figure S4D, E in Supplement 1). Despite the surprising results with 4-EPS, many of the metabolites with the highest fold change and lowest $p$ value are indeed other phenolic molecules, such as metabolites of hippurate, tyrosine, and diet-derived phenols, some of which have been similarly measured in a previous study transferring fecal microbes from individuals with ASD into mice (Figure S4F in Supplement 1; Table S10 in Supplement 2) (73). While preliminary, these studies reveal dysregulated microbiome-mediated effects on xenobiotic pathways and phenolic metabolites in ASD. 

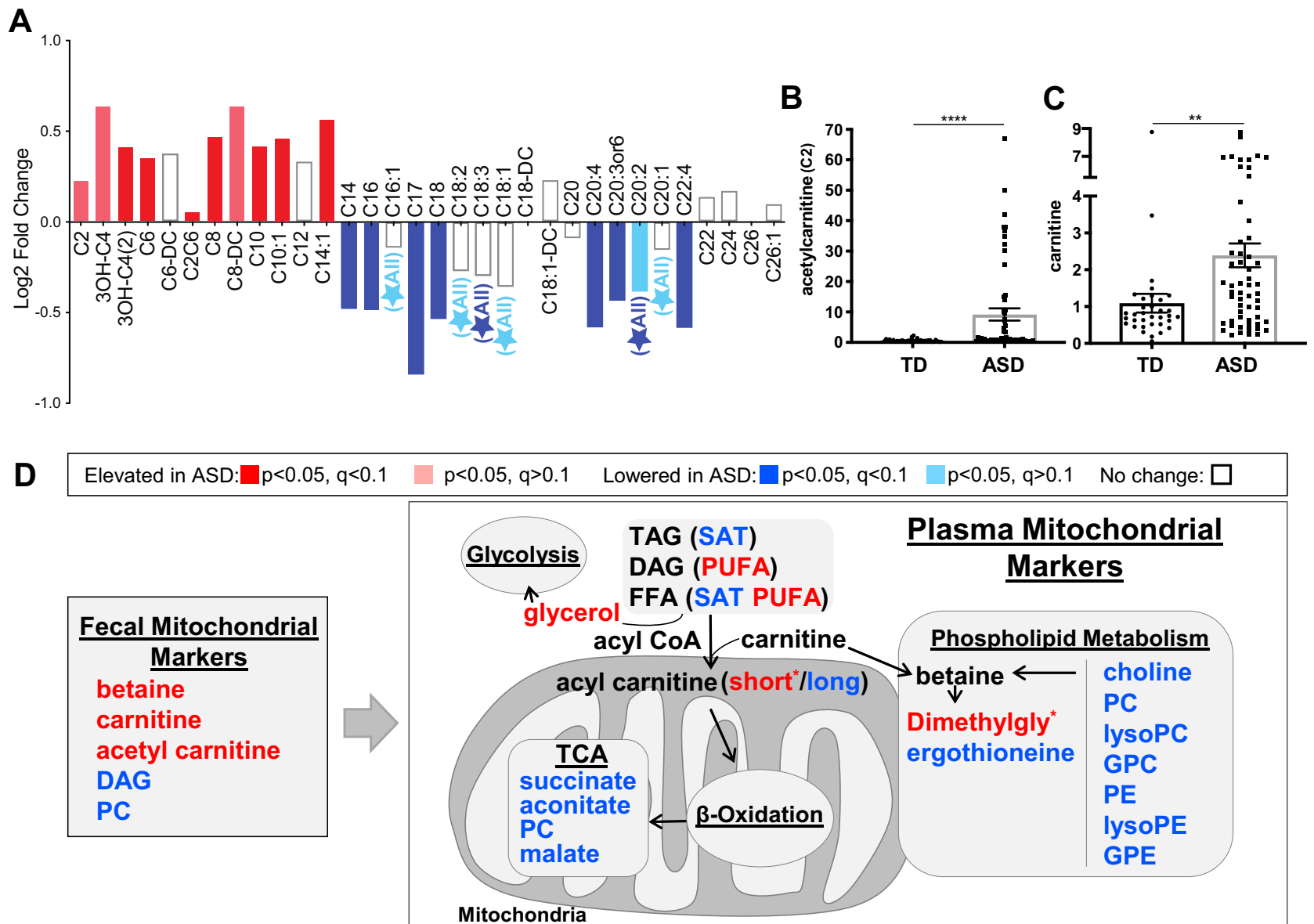

Figure 3. Autism spectrum disorder (ASD) abnormalities within cellular energy and oxidative stress metabolites. (A) Log 2 fold change of acylcarnitines in the plasma of ASD samples without gastrointestinal symptoms compared with typically developing (TD) control samples. Significance indicated by color according to legend below, determined by analysis of variance contrasts. Star indicates that a trend or significance was observed but only in the comparison between all samples. (B, C) Scaled intensity values indicating relative levels of acetylcarnitine (C2) and carnitine in ASD fecal samples compared with TD control samples (all samples). Data are represented as mean \pm SEM. Asterisks indicate significance in analysis of variance contrasts (false discovery rate cutoff $q<.1$ ) performed on total metabolomics dataset $\left({ }^{\star \star} p<.01,{ }^{\star \star \star \star} p<.0001\right)$. (D) Schematic of mitochondrial markers and other metabolites closely associated with cellular energy in plasma (within center box) and feces (boxed to left). *Significant only in ASD samples without gastrointestinal symptoms. Color of text indicates direction and significance of change according to legend above. DAG, diacylglycerol; FFA, free fatty acid; GPC, glycerophosphocholine; GPE, glycerophosphoethanolamine; GPG, glycerophosphoglycerol; GPI, glycerophosphoinositol; GPS, glycerophosphoserine; PC, phosphatidylcholine; PE, phosphatidylethanolamine; PUFA, polyunsaturated fatty acid; SAT, saturated fatty acid; TAG, triacylglycerol.

\section{Steroid Hormone Levels Are Elevated in ASD}

Multiple human ASD studies have examined the levels of specific steroid metabolites within androgenic, pregnenolone, and progesterone metabolism, with some finding aberrant levels, positive correlations with ASD severity, and behavioral improvement following treatments that lower levels of certain hormones $(49,65,74-81)$. In contrast, in a recent clinical trial of children with ASD given an antioxidant treatment, levels of pregnenolones and androgens increased and correlated with improved behavior (54). In our dataset, we found increases of many detected metabolites within the pregnenolone and androgen pathways (Figures 1C, 4A; Table S1 in Supplement 2). This is an indicator that the physiological pathways associated with the downstream metabolism of cholesterol are significantly altered between ASD and TD populations. There does not appear to be a global change in steroid metabolism, as most primary bile acid and sterol metabolites were unaffected (Tables S1 and S4 in Supplement 2). We observed some elevation of these hormone levels independent of sex, which is notable considering the male bias in ASD, reflected in our primarily male sample set $(7 \%-15 \%$ female) (Figure S6A, B in Supplement 1) (82). Because a cluster of our samples are from older individuals in the ASD group, and to account for agedependent increases in androgens (Table S7 in Supplement 2), we stratified by age and still observed heightened androgenic and pregnenolone metabolite levels in ASD subpopulations (Figure S6C in Supplement 1). Taken together, these data indicate that steroidal hormone metabolism may be altered in the ASD population relative to TD samples and that these differences are not driven solely by sex or age differences in our cohort. 


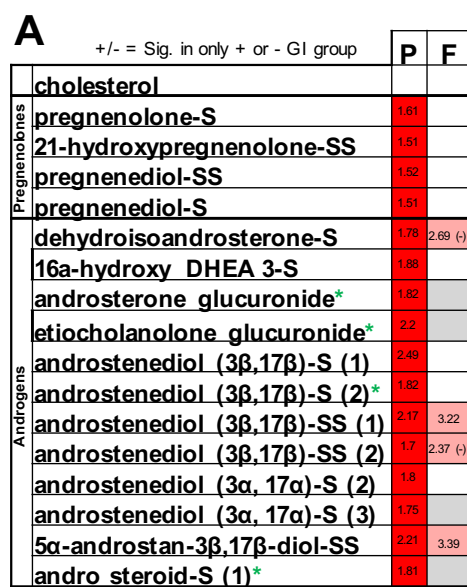

andro steroid-S (1)

*detected in $<70 \%$ of samples
B

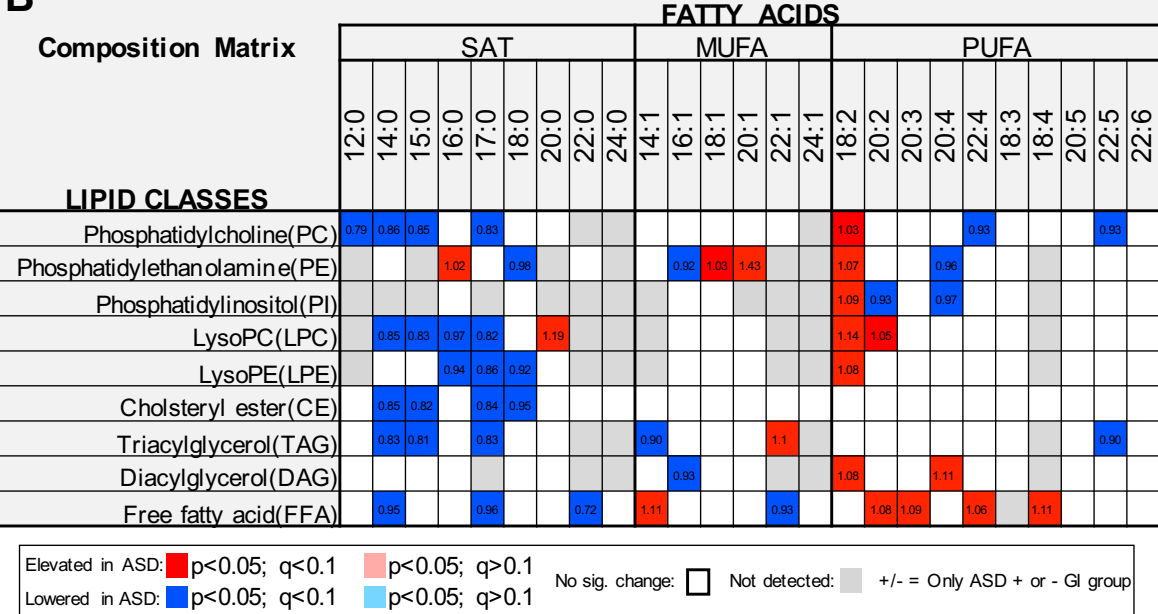

Figure 4. Steroid hormone levels are elevated in autism spectrum disorder (ASD), and other lipid metabolite levels differ in ASD. (A) Significant alterations to levels of all metabolites are detected in the pregnenolone, progestin, and androgen steroid pathways in plasma $(P)$ and feces $(F)$, with colors indicating significance and fold change direction according to legend at bottom right and numerical fold change in text within the box. (B) Complex lipid panel results for all ASD plasma samples compared with typically developing control samples with acyl chain length of lipids across the top, described by chain length and degree of unsaturation and categorized by saturated (SAT), monounsaturated (MUFA), and polyunsaturated (PUFA) fatty acids. Lipid classes are listed along the left. Direction of change and significance are indicated by the legend. Significance determined by analysis of variance contrasts; false discovery rate cutoff $q<$.1. DHEA, dehydroepiandrosterone; Gl, gastrointestinal (symptoms); sig., significant.

\section{Lipid Metabolite Levels Differ in ASD}

Lipids are crucial for energy storage, cellular membrane integrity, and cell signaling. They play a variety of roles in the central nervous system, and their dysregulation has been linked to ASD $(11,13)$. Phospholipids have been measured at lower levels, while long-chain fatty acids are reportedly elevated, but polyunsaturated fatty acids (PUFAs) have been measured at both higher and lower levels, depending on the cohort $(11,14,15)$. Here, we performed a comprehensive, quantitative metabolite analysis on complex lipids. The concentrations of 999 lipids were quantified, and $13.7 \%$ of these were significantly different in the ASD samples, with $4.6 \%$ increased and $9.1 \%$ decreased compared with TD samples. Many of these differentially abundant lipids included phospholipids, cholesterol esters, and glycerolipids (Figure S7A in Supplement 1). In general, shorter (14-18 chain length) saturated fatty acids were less abundant in ASD throughout the lipid classes (Figure 4C; Figure S7B in Supplement 1).

PUFA lipid levels were also different, with elevations in diacylglycerols and free fatty acids and an enrichment of the 18:2 (linolenic) chain length in most lipid classes (Figure 4C). Fecal samples generally trended in the opposite direction from plasma in PUFA lipids (Figure S7C in Supplement 1; Table S4 in Supplement 2). PUFA lipids containing linolenic acids are precursors to other PUFAs (arachidonic and docosahexaenoic acids) important for brain development, function, and structural integrity (14). Future studies are needed to determine if these changes in lipid levels contribute to ASD.

Considering the prevalence of intestinal issues in ASD, GIdependent metabolite analysis between individuals may provide useful insights. Accordingly, we found a total of 87 and 24 metabolites in plasma and feces, respectively, that were potentially differential within $\mathrm{ASD}+\mathrm{Gl}$ compared with ASD-GI individuals by analysis of variance contrasts, but none passed the false discovery rate cutoff (Figure S5A, B in Supplement 1). At the pathway level, we found enrichments in free fatty acids between ASD + Gl and ASD-GI plasma samples (Figure S5C in Supplement 1), but none in fecal samples (Table $S 4$ in Supplement 2). In plasma, free fatty acids of multiple chain lengths trended lower in ASD+Gl compared with ASD-Gl samples (Figure S5D in Supplement 1). Some fatty acids, such as PUFAs, are anti-inflammatory, and their lower levels may contribute to Gl dysfunction directly (14). We did not observe significant correlations between lipid (or other) metabolite patterns and Gl symptoms or disease, including diarrhea, constipation, or irritable bowel syndrome. While these results are interesting, the implications of correlating an altered metabolome and $\mathrm{Gl}$ symptoms in ASD remain to be determined.

\section{Differential Phenolic Xenobiotic Metabolite Levels in ASD}

Phenolic metabolites, a diverse structural class of thousands of molecules containing a phenol moiety, come from dietary ingredients or biotransformation of aromatic amino acids by the gut microbiota. In their free phenolic forms, they can be readily absorbed through intestinal tissues; however, microbial modification of these molecules can significantly alter their absorption, bioavailability, and bioactivity (83), leading to various benefits or harm to the host $(83,84)$. In fact, altered levels of phenolics have been highlighted in many ASD metabolomic studies $(20,22-28,30,33,34,54,71,85,86)$. However, a consensus of these metabolite changes in ASD has yet 
A

\begin{tabular}{|c|c|c|}
\hline Sig. Altered Metabolite & $\mathbf{P}$ & $\mathbf{F}$ \\
\hline 4-ethylphenyl sulfate & 6.89 & \\
\hline 3-hydroxybenzoate & & 0.7 \\
\hline 2-ethylphenyl sulfate & 0.72 & \\
\hline phenol sulfate & 0.84 & \\
\hline 3-(4-hydroxyphenyl)lactate & 0.9 & \\
\hline 4-allylphenol sulfate & 0.72 & \\
\hline salicyluric glucuronide & 0.88 & \\
\hline 2-aminophenol sulfate & 0.67 & \\
\hline 4-methylbenzenesulfonate* & 59.6 & \\
\hline genistein sulfate* & $5.44(+)$ & \\
\hline equol glucuronide* & $2.0(+)$ & \\
\hline equol sulfate* & $2.04(+)$ & \\
\hline daidzein sulfate $(2)^{\star}$ & 3.41 & \\
\hline salicylate & 0.89 & 0.49 \\
\hline 3-hydroxyhippurate & 0.77 & \\
\hline caffeic acid sulfate & $0.62(-)$ & \\
\hline hydroquinone sulfate & 0.72 & \\
\hline 4-hydroxyhippurate & 0.86 & \\
\hline isoeugenol sulfate* & $0.46+$ & \\
\hline 2-hydroxyhippurate & 0.89 & \\
\hline o-cresol sulfate* & 0.58 & \\
\hline vanillactate* & 0.82 & \\
\hline vanillic alcohol sulfate & 0.63 & \\
\hline 4-hydroxycoumarin & 0.86 & \\
\hline m-tyramine* & & 5.4 \\
\hline 4-hydroxybenzoate & & $0.51+$ \\
\hline 4-methylcatechol sulfate* & & 0.61 \\
\hline p-cresol sulfate & & 0.48 \\
\hline gentisate & & 0.75 \\
\hline 3-hydroxyphenylacetate & & 0.49 \\
\hline dihydroferulic acid & & 0.5 \\
\hline sinapate & & 0.34 \\
\hline \multicolumn{3}{|l|}{ ASD/TD } \\
\hline \multicolumn{3}{|c|}{ 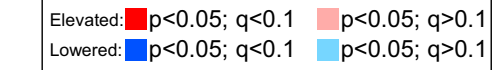 } \\
\hline \multicolumn{3}{|c|}{ No sig. change: $\square \quad$ Not detected: $\square$} \\
\hline \multicolumn{3}{|c|}{$+/==$ Only ASD + or - Gl group } \\
\hline
\end{tabular}

B

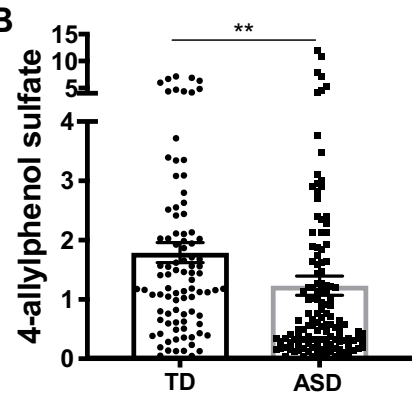

C $\left.{ }_{0}^{3}\right]$

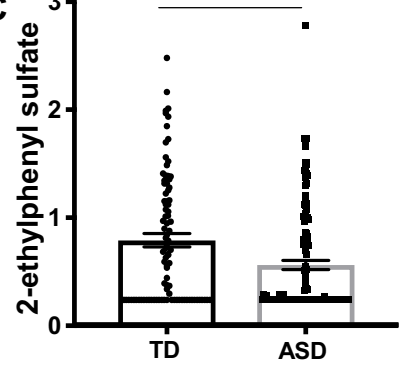

D
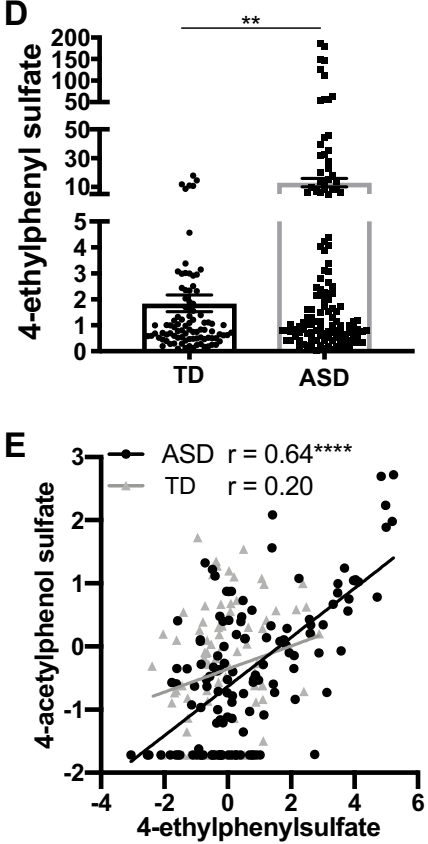

Figure 5. Differential phenolic xenobiotic metabolite levels in autism spectrum disorder (ASD). (A) Phenolic metabolites belonging to the benzoate, tyrosine, and food component/plant pathways that are significantly different between ASD vs. typically developing (TD) groups in plasma $(P)$ and feces $(F)$. Directionality and significance defined in legend, and numerical fold change in text within the box. (B) Scaled intensity values indicating relative levels of 4-allylphenol sulfate in plasma (all samples). (C) Scaled intensity values indicating relative levels of 2-ethylphenyl sulfate in plasma (all samples). (D) Scaled intensity values indicating relative levels of 4-ethylphenyl sulfate in plasma (all samples). (E) Spearman correlation between plasma 4-ethylphenyl sulfate and 4-acetylphenol sulfate, $\log _{2}$ scale. Data in panels (B-D) are represented as mean \pm SEM. Asterisks indicate significance in analysis of variance contrasts with a false discovery rate cutoff $q<.1$ performed on total metabolomics dataset $\left.{ }^{* \star} p<.01,{ }^{\star \star \star \star} p<.0001\right)$. Gl, gastrointestinal (symptoms); sig., significantly. to be reached, and most studies have measured only a subset of these metabolites.

We observed altered or trending levels of phenolic metabolites belonging to several interrelated pathways, including tyrosine, benzoate, and food component/plant metabolites (Figure 5A). Some share structural similarity to para-cresol sulfate, a toxic molecule elevated in the urine of young children with ASD $(22,24)$. These include, among others, 4-EPS, 2ethylphenylsulfate, cresol derivatives, 4-allylphenyl sulfate, and 4-methylbenzenesulfonate; the last-mentioned was elevated a remarkable 60 -fold in the small subset of ASD samples in which it was detected (Figure 5A-D; Table S1 in Supplement 2). Changes in the levels of these phenolic molecules have also been observed in animal models of ASD $(52,87)$. Previously, 4EPS was observed with a 46-fold elevation in the maternal immune activation mouse model, and administration of synthetic 4-EPS to wild-type mice was sufficient to induce an anxiety-like phenotype (52). Here, in ASD plasma samples, 4-EPS levels were increased 6.9-fold (Figure 5D). This was largely driven by a considerable subpopulation of individuals with ASD with high 


\begin{tabular}{|c|c|c|}
\hline & Metabolite(s) & Core Findings \\
\hline \multirow{4}{*}{$\frac{n}{\frac{0}{2}}$} & Steroid hormones & $\begin{array}{c}\text { Pathways enriched; pregnenolone and androgen } \\
\text { hormone metabolite levels elevated }\end{array}$ \\
\hline & PUFAs & $\begin{array}{c}\begin{array}{c}\text { Elevated levels in DAG, FFA, and many with chain } \\
\text { length 18:2 }\end{array} \\
\end{array}$ \\
\hline & Saturated FAs & Pathways enriched; broad decrease in levels \\
\hline & Free FAs & Trending decreased levels in ASD+GI \\
\hline \multirow{4}{*}{ 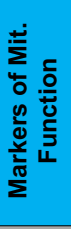 } & Acyl-carnitines & $\begin{array}{l}\text { Pathways enriched; shorter chain moieties elevated, } \\
\text { longer chain moieties decreased; acetyl- and free } \\
\text { carnitine elevated in feces }\end{array}$ \\
\hline & Phospholipid metabolism & Pathway enriched in feces \\
\hline & TCA cycle & Lower levels of succinate, aconitate, malate levels \\
\hline & $\begin{array}{c}\text { Pathways rel. to oxidative stress (Cys, Met., } \\
\text { SAM; glutathione; gg-AA metabolites) }\end{array}$ & Significant correlations with ADOS SS \\
\hline \multirow{3}{*}{ 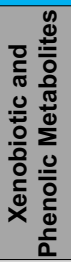 } & $\begin{array}{l}\text { Phenolic structure mets.: 4-ethylphenyl } \\
\text { sulfate (4EPS), genistein-S, equol mets., } \\
\text { daidzein sulfate, 4-methylbenzene sulfonate }\end{array}$ & Elevated to various degrees in ASD plasma samples \\
\hline & $\begin{array}{l}\text { Phenolic structure mets.: hippurate and } \\
\text { benzoic acid mets., some tyrosine mets., } \\
\text { salicylic mets. }\end{array}$ & $\begin{array}{l}\text { Decreased to various degrees in ASD plasma } \\
\text { samples }\end{array}$ \\
\hline & $\begin{array}{c}\text { Phenolic structure mets.: equol-S, 3- } \\
\text { phenylpropionate, hippurate, 4- } \\
\text { hydroxyhippurate, caffeic acid-S, phenol-S, } \\
\text { daidzein-S, genistein-S }\end{array}$ & $\begin{array}{l}\text { Display shifted levels in mouse recipients of human } \\
\text { ASD fecal samples compared to TD fecal samples }\end{array}$ \\
\hline \multirow{4}{*}{ 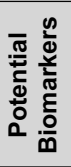 } & Acyl-carnitines and lipids & $\begin{array}{c}\text { Rank in the top } 30 \text { most discriminatory metabolites, } \\
\text { assessed by Random Forest analysis }\end{array}$ \\
\hline & $\begin{array}{l}\text { Metabolites known to be influenced by the } \\
\text { gut microbiota }\end{array}$ & $\begin{array}{c}\text { Rank in the top } 30 \text { most discriminatory metabolites, } \\
\text { assessed by Random Forest analysis }\end{array}$ \\
\hline & Indolelactate, margaroylcarnitine & Most discriminatory plasma metabolites \\
\hline & Nicotinamide, 9-HOTrE & Most discriminatory fecal metabolites \\
\hline
\end{tabular}

Figure 6. Summary chart of core findings. Categorized into metabolites of lipid, mitochondrial function marker, and xenobiotic and phenolic pathways as well as potential biomarkers, the metabolites of most interest (left) and observations made from the data (right) are summarized. ADOS, Autism Diagnostic Observation Schedule; ASD, autism spectrum disorder; Cys, cysteine; DAG, diacylglycerol; FAs, fatty acids; FFA, free fatty acid; ggAA, gamma-glutamyl amino acids; Gl, gastrointestinal (symptoms); Met., methionine; mets., metabolites; Mit., mitochondrial; PUFA, polyunsaturated fatty acid; SAM, S-adenosyl-L-methionine; SS, severity score; TCA, tricarboxylic acid; TD, typically developing. levels of 4-EPS, who also show higher levels of variation in related phenolic metabolites (Figure S8A in Supplement 1). In many cases, phenolic metabolite levels correlate with 4-EPS in ASD samples, including 4-acetylphenyl sulfate, a derivative of 4EPS, and others (Figure 5E; Figure S8B, S8C in Supplement 1). These observed alterations, combined with mounting evidence from previous studies, suggest that phenolic metabolites may be involved in ASD.

\section{DISCUSSION}

Changes in the metabolome have been linked to a number of neurodevelopmental and neurodegenerative disorders $(88,89)$. The current study includes a comprehensive profiling of the metabolites from 231 plasma and 97 matched fecal samples from individuals with ASD and TD who underwent extensive behavioral testing. We observed that specific individual metabolites and metabolic pathways in lipid, amino acid, and xenobiotic metabolism were altered between ASD and TD groups and that many of these same molecules and pathways correlated to severity of ASD behavioral scores. The core findings are summarized in Figure 6. Various steroid hormone metabolite levels were elevated in ASD samples. PUFA levels and short-chain acylcarnitines were generally elevated in ASD plasma samples, while saturated fatty acid levels and longchain acylcarnitines were decreased, contributing to a general picture of dysregulated cellular energy and oxidative state, with potential connections to mitochondrial dysfunction in ASD. Some of the lipid and xenobiotic metabolites also showed interesting changes within the ASD group when stratified by the presence of Gl symptoms. Finally, many phenolic metabolites derived largely from host and bacterial metabolism of amino acids, plant polyphenols, and other food components were detected at differential levels in plasma and feces between groups.

ASD is diagnosed by behavioral tests, with extensive heterogeneity of symptoms, severity, and etiology between individuals and little consensus on molecular mechanisms. This enigmatic spectrum has a strong but complex genetic basis, with hundreds of reported risk genes (90). The contributions of risk alleles to behavior is a vast and active area of research. In addition to genetics, understanding of altered metabolite levels in blood, feces, and brains of individuals with ASD may provide a glimpse into physiological aspects of the disorder and hold the potential to advance diagnosis and/or stratification of subpopulations of ASD. In fact, our results broadly support the notion of multiple etiologies or endophenotypes within the ASD population.

Interestingly, multiple mouse models support the notion that gut metabolites are associated with brain development and function $(50,91,92)$. Some metabolites are closely linked with neurological disorders, either to positive or to negative outcomes $(93,94)$. Several examples have been reported of gut metabolites entering circulation and directly affecting the brain, as well as cases where metabolites stimulate pathways in the gut, immune, or autonomic nervous system and exert changes to the brain and to behavior (95-98). Comprehensive metabolic profiling in humans and animal models provides insight into the molecular status of disease and how genetic factors and environmental influences (dietary, microbial, and chemical) interact. Deeper analysis of our dataset along with additional studies, with future empirical studies to validate the relevance of our observations, could illuminate aspects of ASD pathophysiology. The intriguing correlations between ASD behaviors, altered levels of fecal and plasma metabolites, and GI 
symptoms contribute to the concept that ASD may be viewed as a whole-body condition and argue for increased investigation into peripheral aspects of disease that may lead to advances in diagnosis and improved stratification of ASD populations.

\section{ACKNOWLEDGMENTS AND DISCLOSURES}

This work was supported by Autism Speaks (Grant No. 7567 [to PA, AF, and SKM]), the Johnson Foundation (to PA), the Brain Foundation (to PA), Genome, Environment, Microbiome, and Metabolome in Autism (Grant No. 825033 [to AF]), Axial Biotherapeutics (to SKM), and the National Institutes of Health (Grant No. HD090214 [to PA] and Grant No. MH100556 [to SKM]).

We thank the MIND Institute study staff, and the dedication and commitment of the families who took part in these studies are gratefully acknowledged.

ASC, DHD, and SKM have financial interest in Axial Biotherapeutics. AF has financial interest in Alba Therapeutics. GMP and MCC are employed by Axial Biotherapeutics. All other authors report no biomedical financial interests or potential conflicts of interest.

\section{ARTICLE INFORMATION}

From the Division of Biology and Biological Engineering (BDN, MDA, SKM) California Institute of Technology, Pasadena; Department of Medical Microbiology and Immunology (DRR, PA), University of California, Davis, Davis; MIND Institute (DRR, PA), University of California, Davis, Sacramento, California; Division of Pediatric Gastroenterology and Nutrition (GS, AF), Mucosal Immunology and Biology Research Center, Massachusetts General Hospital for Children, Boston; and Axial Biotherapeutics (GMP, MCC, ASC, DHD), Waltham, Massachusetts.

Address correspondence to Sarkis K. Mazmanian, Ph.D., at sarkis@ caltech.edu, or Brittany D. Needham, Ph.D., at bneedham@caltech.edu.

Received Apr 15, 2020; revised and accepted Sep 30, 2020.

Supplementary material cited in this article is available online at https:// doi.org/10.1016/j.biopsych.2020.09.025.

\section{REFERENCES}

1. Doernberg E, Hollander E (2016): Neurodevelopmental disorders (ASD and ADHD): DSM-5, ICD-10, and ICD-11. CNS Spectr 21:295-299.

2. Baio J, Wiggins L, Christensen DL, Maenner MJ, Daniels J, Warren Z, et al. (2018): Prevalence of autism spectrum disorder among children aged 8 years-autism and developmental disabilities monitoring network, 11 sites, United States, 2014. MMWR Surveill Summ 67:123.

3. Dawson G, Rogers S, Munson J, Smith M, Winter J, Greenson J, et al. (2010): Randomized, controlled trial of an intervention for toddlers with autism: The Early Start Denver Model. Pediatrics 125:e17-e23.

4. Virués-Ortega J (2010): Applied behavior analytic intervention for autism in early childhood: Meta-analysis, meta-regression and doseresponse meta-analysis of multiple outcomes. Clin Psychol Rev 30:387-399.

5. Chiarotti F, Venerosi A (2020): Epidemiology of autism spectrum disorders: A review of worldwide prevalence estimates since 2014. Brain Sci 10:274.

6. Elsabbagh M, Divan G, Koh YJ, Kim YS, Kauchali S, Marcín C, et al. (2012): Global prevalence of autism and other pervasive developmental disorders. Autism Res 5:160-179.

7. Azhari A, Azizan F, Esposito G (2019): A systematic review of gutimmune-brain mechanisms in autism spectrum disorder. Dev Psychobiol 61:752-771.

8. Rossignol DA, Frye RE (2012): Mitochondrial dysfunction in autism spectrum disorders: A systematic review and meta-analysis. Mol Psychiatry 17:290-314.

9. Cheng N, Rho JM, Masino SA (2017): Metabolic dysfunction underlying autism spectrum disorder and potential treatment approaches. Front Mol Neurosci 10:34.
10. El-Ansary A, Al-Ayadhi L (2012): Lipid mediators in plasma of autism spectrum disorders. Lipids Health Dis 11:160.

11. El-Ansary AK, Bacha AGB, Al-Ayahdi LY (2011): Impaired plasma phospholipids and relative amounts of essential polyunsaturated fatty acids in autistic patients from Saudi Arabia. Lipids Health Dis 10:63.

12. James SJ, Cutler P, Melnyk S, Jernigan S, Janak L, Gaylor DW Neubrander JA (2004): Metabolic biomarkers of increased oxidative stress and impaired methylation capacity in children with autism. Am J Clin Nutr 80:1611-1617.

13. Lv QQ, You C, Zou XB, Deng HZ (2018): Acyl-carnitine, C5DC, and C26 as potential biomarkers for diagnosis of autism spectrum disorder in children. Psychiatry Res 267:277-280.

14. Mostafa GA, Al-Ayadhi LY (2015): Reduced levels of plasma polyunsaturated fatty acids and serum carnitine in autistic children: Relation to gastrointestinal manifestations. Behav Brain Funct 11:4.

15. Pastural E, Ritchie S, Lu Y, Jin W, Kavianpour A, Khine Su-Myat K, et al. (2009): Novel plasma phospholipid biomarkers of autism: mitochondrial dysfunction as a putative causative mechanism. Prostaglandins Leukot Essent Fatty Acids 81:253-264.

16. Wang H, Liang S, Wang M, Gao J, Sun C, Wang J, et al. (2016): Potential serum biomarkers from a metabolomics study of autism. J Psychiatry Neurosci 41:27-37.

17. West PR, Amaral DG, Bais P, Smith AM, Egnash LA, Ross ME, et al. (2014): Metabolomics as a tool for discovery of biomarkers of autism spectrum disorder in the blood plasma of children. PLoS One 9 e112445.

18. Cozzolino R, De Magistris L, Saggese P, Stocchero M, Martignetti A Di Stasio M, et al. (2014): Use of solid-phase microextraction coupled to gas chromatography-mass spectrometry for determination of urinary volatile organic compounds in autistic children compared with healthy controls. Anal Bioanal Chem 406:4649-4662.

19. Ming X, Stein TP, Barnes V, Rhodes N, Guo L (2012): Metabolic perturbance in autism spectrum disorders: A metabolomics study. J Proteome Res 11:5856-5862.

20. Noto A, Fanos V, Barberini L, Grapov D, Fattuoni C, Zaffanello M, et al. (2014): The urinary metabolomics profile of an Italian autistic children population and their unaffected siblings. J Matern Fetal Neonatal Med 27(Suppl 2):46-52.

21. Shaw W, Kassen E, Chaves E (1995): Increased urinary excretion of analogs of Krebs cycle metabolites and arabinose in two brothers with autistic features. Clin Chem 41:1094-1104.

22. Altieri L, Neri C, Sacco R, Curatol P, Benvenuto A, Muratori F, et al. (2011): Urinary p-cresol is elevated in small children with severe autism spectrum disorder. Biomarkers 16:252-260.

23. Emond P, Mavel S, Aïdoud N, Nadal-Desbarats L, Montigny F, Bonnet-Brilhault F, et al. (2013): GC-MS-based urine metabolic profiling of autism spectrum disorders. Anal Bioanal Chem 405:5291-5300.

24. Gabriele S, Sacco R, Cerullo S, Neri C, Urbani A, Tripi G, et al. (2014): Urinary $\mathrm{p}$-cresol is elevated in young French children with autism spectrum disorder: A replication study. Biomarkers 19:463-470.

25. Lis AW, Mclaughlin I, Mclaughlin RK, Lis EW, Stubbs EG (1976): Profiles of ultraviolet-absorbing components of urine from autistic children, as obtained by high-resolution ion-exchange chromatography. Clin Chem 22:1528-1532.

26. Lussu M, Noto A, Masili A, Rinaldi AC, Dessì A, Angelis MD, et al. (2017): The urinary ${ }^{1} \mathrm{H}-\mathrm{NMR}$ metabolomics profile of an Italian autistic children population and their unaffected siblings. Autism Res 10:10581066.

27. Nadal-Desbarats L, Aïdoud N, Emond P, Blasco H, Filipiak I, Sarda P, et al. (2014): Combined ${ }^{1} \mathrm{H}-\mathrm{NMR}$ and ${ }^{1} \mathrm{H}-{ }^{13} \mathrm{C}$ HSQC-NMR to improve urinary screening in autism spectrum disorders. Analyst 139:34603468.

28. Yap IKS, Angley M, Veselkov KA, Holmes E, Lindon JC, Nicholson JK (2010): Urinary metabolic phenotyping differentiates children with autism from their unaffected siblings and age-matched controls. J Proteome Res 9:2996-3004.

29. Adams JB, Johansen LJ, Powell LD, Quig D, Rubin RA (2011) Gastrointestinal flora and gastrointestinal status in children with autism-comparisons to typical children and correlation with autism severity. BMC Gastroenterol 11:22. 
30. Angelis MD, Piccolo M, Vannini L, Siragusa S, Giacomo AD, Serrazzanetti DI, et al. (2013): Fecal microbiota and metabolome of children with autism and pervasive developmental disorder not otherwise specified. PLoS One 8:e76993.

31. Kang DW, Ilhan ZE, Isern NG, Hoyt DW, Howsmon DP, Shaffer M, et al. (2018): Differences in fecal microbial metabolites and microbiota of children with autism spectrum disorders. Anaerobe 49:121-131.

32. Wang L, Christophersen CT, Sorich MJ, Gerber JP, Angley MT, Conlon MA (2012): Elevated fecal short chain fatty acid and ammonia concentrations in children with autism spectrum disorder. Dig Dis Sci 57:2096-2102.

33. Bitar T, Mavel S, Emond P, Nadal-Desbarats L, Lefèvre A, Mattar $H$, et al. (2018): Identification of metabolic pathway disturbances using multimodal metabolomics in autistic disorders in a Middle Eastern population. J Pharm Biomed Anal 152:57-65.

34. Gevi F, Zolla L, Gabriele S, Persico AM (2016): Urinary metabolomics of young Italian autistic children supports abnormal tryptophan and purine metabolism. Mol Autism 7:47.

35. Kuwabara H, Yamasue H, Koike S, Inoue H, Kawakubo Y, Kuroda M, et al. (2013): Altered metabolites in the plasma of autism spectrum disorder: A capillary electrophoresis time-of-flight mass spectroscopy study. PLoS One 8:e73814.

36. Orozco JS, Hertz-Picciotto I, Abbeduto L, Slupsky CM (2019): Metabolomics analysis of children with autism, idiopathicdevelopmental delays, and Down syndrome. Transl Psychiatry 9:243.

37. Ferguson BJ, Dovgan K, Severns D, Martin S, Marler S, Gross Margolis K, et al. (2019): Lack of associations between dietary intake and gastrointestinal symptoms in autism spectrum disorder. Front Psychiatry 10:528.

38. Sanctuary MR, Kain JN, Angkustsiri K, German JB (2018): Dietary considerations in autism spectrum disorders: The potential role of protein digestion and microbial putrefaction in the gut-brain axis. Front Nutr 5:40.

39. Cermak SA, Curtin C, Bandini LG (2010): Food selectivity and sensory sensitivity in children with autism spectrum disorders. J Am Diet Assoc 110:238-246.

40. Needham BD, Tang W, Wu WL (2018): Searching for the gut microbial contributing factors to social behavior in rodent models of autism spectrum disorder. Dev Neurobiol 78:474-499.

41. Pulikkan J, Mazumder A, Grace T (2019): Role of the gut microbiome in autism spectrum disorders. Adv Exp Med Biol 1118:253-269.

42. Vuong HE, Yano JM, Fung TC, Hsiao EY (2017): The microbiome and host behavior. Annu Rev Neurosci 40:21-49.

43. Hertz-Picciott I, Croen LA, Hansen R, Jones CR, van de Water J, Pessah IN (2006): The CHARGE Study: An epidemiologic investigation of genetic and environmental factors contributing to autism. Environ Health Perspect 114:1119-1125.

44. Rose DR, Yang H, Serena G, Sturgeon C, Ma B, Careaga M, et al. (2018): Differential immune responses and microbiota profiles in children with autism spectrum disorders and co-morbid gastrointestinal symptoms. Brain Behav Immun 70:354-368.

45. Drossman DA (2006): The functional gastrointestinal disorders and the Rome III process. Gastroenterology 130:1377-1390.

46. Kelly RS, Boulin A, Laranjo N, Lee-Sarwar K, Chu SH, Yadama AP, et al. (2019): Metabolomics and communication skills development in children: Evidence from the Ages and Stages Questionnaire. Metabolites 9:42.

47. Weiler A, Volkenhoff A, Hertenstein H, Schirmeier S (2017): Metabolite transport across the mammalian and insect brain diffusion barriers. Neurobiol Dis 107:15-31.

48. Frye RE, Melnyk S, MacFabe DF (2013): Unique acyl-carnitine profiles are potential biomarkers for acquired mitochondrial disease in autism spectrum disorder. Transl Psychiatry 3:e220.

49. Gillberg C, Fernell E, Kočovská E, Minnis H, Bourgeron T, Thompson L, Allely CS (2017): The role of cholesterol metabolism and various steroid abnormalities in autism spectrum disorders: A hypothesis paper. Autism Res 10:1022-1044.

50. Golubeva AV, Joyce SA, Moloney G, Burokas A, Sherwi E, Arboley S, et al. (2017): Microbiota-related changes in bile acid and tryptophan metabolism are associated with gastrointestinal dysfunction in a mouse model of autism. EBioMedicine 24:166-178.

51. Kurochkin I, Khrameeva E, Tkachev A, Stepanova V, Vanyushkina A, Stekolshchikova E, et al. (2019): Metabolome signature of autism in the human prefrontal cortex. Commun Biol 2:234.

52. Hsiao EY, McBride SW, Hsien S, Sharon G, Hyde ER, McCue T, et al. (2013): Microbiota modulate behavioral and physiological abnormalities associated with neurodevelopmental disorders. Cell 155:14511463.

53. Roager HM, Licht TR (2018): Microbial tryptophan catabolites in health and disease. Nat Commun 9:1-10.

54. Bent S, Lawton B, Warren T, Widjaja F, Dang K, Fahey JW, et al. (2018): Identification of urinary metabolites that correlate with clinical improvements in children with autism treated with sulforaphane from broccoli. Mol Autism 9:35.

55. Mavel S, Nadal-Desbarats L, Blasco H, Bonnet-Brilhault F, Barthélémy C, Montigny $\mathrm{F}$, et al. (2013): ${ }^{1} \mathrm{H}-{ }^{13} \mathrm{C}$ NMR-based urine metabolic profiling in autism spectrum disorders. Talanta 114:95-102.

56. Ripps H, Shen W (2012): Review: Taurine: A "very essential" amino acid. Mol Vis 18:2673-2686.

57. Dickinson A, Jones M, Milne E (2016): Measuring neural excitation and inhibition in autism: Different approaches, different findings and different interpretations. Brain Res 1648:277-289.

58. Zheng HF, Wang WQ, Li XM, Rauw G, Bake GB (2017): Body fluid levels of neuroactive amino acids in autism spectrum disorders: A review of the literature. Amino Acids 49:57-65.

59. Smith AM, King JJ, West PR, Ludwig MA, Donley ELR, Burrier RE, Amaral DG (2019): Amino acid dysregulation metabotypes: Potential biomarkers for diagnosis and individualized treatment for subtypes of autism spectrum disorder. Biol Psychiatry 85:345-354.

60. Olson CA, Vuong HE, Yano JM, Liang QY, Nusbaum DJ, Hsiao EY (2018): The gut microbiota mediates the anti-seizure effects of the ketogenic diet. Cell 173:1728-1741.e13.

61. Liu A, Zhou W, Qu L, He F, Wang H, Wang Y, et al. (2019): Altered urinary amino acids in children with autism spectrum disorders. Front Cell Neurosci 13:7.

62. Chang CY, Ke DS, Chen JY (2009): Essential fatty acids and human brain. Acta Neurol Taiwan 18:231-241.

63. Kuzawa CW, Chugani HT, Grossman LI, Lipovich L, Muzik O, Hof PR, et al. (2014): Metabolic costs and evolutionary implications of human brain development. Proc Natl Acad Sci U S A 111:13010-13015.

64. O'Brien JS, Sampson EL (1965): Lipid composition of the normal human brain: Gray matter, white matter, and myelin. J Lipid Res 6:537-544.

65. Hassan MH, Desoky T, Sakhr HM, Gabra RH, Bakri AH (2019): Possible metabolic alterations among autistic male children: Clinical and biochemical approaches. J Mol Neurosci 67:204-216.

66. Griffiths KK, Levy RJ (2017): Evidence of mitochondrial dysfunction in autism: Biochemical links, genetic-based associations, and nonenergy-related mechanisms. Oxid Med Cell Longev 2017:4314025.

67. Legido A, Jethva R, Goldenthal MJ (2013): Mitochondrial dysfunction in autism. Semin Pediatr Neurol 20:163-175.

68. Lombard J (1998): Autism: A mitochondrial disorder? Med Hypotheses 50:497-500.

69. Coleman M, Blass JP (1985): Autism and lactic acidosis. J Autism Dev Disord 15:1-8.

70. MacFabe DF (2015): Enteric short-chain fatty acids: Microbial messengers of metabolism, mitochondria, and mind: Implications in autism spectrum disorders. Microb Ecol Health Dis 26:28177.

71. Diémé B, Mavel S, Blasco H, Tripi G, Bonnet-Brilhault F, Malvy J, et al. (2015): Metabolomics study of urine in autism spectrum disorders using a multiplatform analytical methodology. J Proteome Res 14:5273-5282.

72. Santamaría L, Reverón I, de Felipe FL, de las Rivas B, Muñoz R (2018): Ethylphenol formation by Lactobacillus plantarum: Identification of the enzyme involved in the reduction of vinylphenols. Appl Environ Microbiol 84.. e01064-18.

73. Sharon G, Cruz NJ, Kang DW, Gandal MJ, Wang B, Kim YM, et al. (2019): Human gut microbiota from autism spectrum disorder promote behavioral symptoms in mice. Cell 177:1600-1618.e17. 
74. Auyeung B, Ahluwalia J, Thomson L, Taylor K, Hackett G, O’Donnell KJ, Baron-Cohen S (2012): Prenatal versus postnatal sex steroid hormone effects on autistic traits in children at 18 to 24 months of age. Mol Autism 3:17.

75. Baron-Cohen S, Auyeung B, Nørgaard-Pedersen B, Hougaard DM, Abdallah MW, Melgaard L, et al. (2015): Elevated fetal steroidogenic activity in autism. Mol Psychiatry 20:369-376.

76. Mamidala MP, Polinedi A, Kumar PT, Rajesh N, Vallamkonda OR, Udani V, et al. (2013): Maternal hormonal interventions as a risk factor for autism spectrum disorder: An epidemiological assessment from India. J Biosci 38:887-892.

77. Whitaker-Azmitia PM, Lobel M, Moyer A (2014): Low maternal progesterone may contribute to both obstetrical complications and autism. Med Hypotheses 82:313-318.

78. Majewska MD, Hill M, Urbanowicz E, Rok-Bujko P, Bieńkowski $P$ Namysłowska I, Mierzejewski P (2014): Marked elevation of adrenal steroids, especially androgens, in saliva of prepubertal autistic children. Eur Child Adolesc Psychiatry 23:485-498.

79. Willing J, Wagner CK (2016): Exposure to the synthetic progestin, $17 \alpha$ hydroxyprogesterone caproate during development impairs cognitive flexibility in adulthood. Endocrinology 157:77-82.

80. Quartier A, Chatrousse L, Redin C, Keime C, Haumesser N, MaglottRoth A, et al. (2018): Genes and pathways regulated by androgens in human neural cells, potential candidates for the male excess in autism spectrum disorder. Biol Psychiatry 84:239-252.

81. Geier DA, Kern JK, King PG, Sykes LK, Geier MR (2012): An evaluation of the role and treatment of elevated male hormones in autism spectrum disorders. Acta Neurobiol Exp (Wars) 72:1-17.

82. Kanner L (1968): Autistic disturbances of affective contact. Acta Paedopsychiatr 35:100-136.

83. Duda-Chodak A, Tarko T, Satora P, Sroka P (2015): Interaction of dietary compounds, especially polyphenols, with the intestinal microbiota: A review. Eur J Nutr 54:325-341.

84. Valdés L, Cuervo A, Salazar N, Ruas-Madiedo P, Gueimonde M, González S (2015): The relationship between phenolic compounds from diet and microbiota: Impact on human health. Food Funct 6:2424-2439.

85. Kałużna-Czaplińska J, Żurawicz E, Struck W, Markuszewski M (2014) Identification of organic acids as potential biomarkers in the urine of autistic children using gas chromatography/mass spectrometry. J Chromatogr B Analyt Technol Biomed Life Sci 966:70-76.
86. Johannsen WJ, Friedman SH, Feldman El, Negrete A (1962): A reexamination of the hippuric acid-anxiety relationship. Psychosom Med 24:569.

87. Naviaux JC, Schuchbauer MA, Li K, Wang L, Risbrough VB, Powell SB, Naviaux RK (2014): Reversal of autism-like behaviors and metabolism in adult mice with single-dose antipurinergic therapy. Transl Psychiatry 4:e400.

88. Grochowska M, Laskus T, Radkowski M (2019): Gut microbiota in neurological disorders. Arch Immunol Ther Exp (Wars) 67:375-383.

89. Sharon G, Sampson TR, Geschwind DH, Mazmanian SK (2016): The central nervous system and the gut microbiome. Cell 167:915-932.

90. lakoucheva LM, Muotri AR, Sebat J (2019): Getting to the cores of autism. Cell 178:1287-1298.

91. Desbonnet L, Clarke G, Traplin A, O'Sullivan O, Crispie F, Moloney RD, et al. (2015): Gut microbiota depletion from early adolescence in mice: Implications for brain and behaviour. Brain Behav Immun 48:165-173.

92. Neufeld KM, Kang N, Bienenstock J, Foster JA (2011): Reduced anxiety-like behavior and central neurochemical change in germ-free mice. Neurogastroenterol Motil 23:255-264, e119.

93. Kujawska M, Jodynis-Liebert J (2018): Polyphenols in Parkinson's disease: A systematic review of in vivo studies. Nutrients 10:642.

94. Sampson TR, Debelius JW, Thron T, Janssen S, Shastri GG, Ilhan ZE, et al. (2016): Gut microbiota regulate motor deficits and neuroinflammation in a model of Parkinson's disease. Cell 167:1469-1480.e12.

95. Jaglin M, Rhimi M, Philippe C, Pons N, Bruneau A, Goustard B, et al. (2018): Indole, a signaling molecule produced by the gut microbiota negatively impacts emotional behaviors in rats. Front Neurosci 12:216.

96. O’Connor JC, Lawson MA, André C, Moreau M, Lestage J, Castanon N, et al. (2009): Lipopolysaccharide-induced depressive-like behavior is mediated by indoleamine 2,3-dioxygenase activation in mice. Mol Psychiatry 14:511-522.

97. Tian P, Wang G, Zhao J, Zhang H, Chen W (2019): Bifidobacterium with the role of 5-hydroxytryptophan synthesis regulation alleviates the symptom of depression and related microbiota dysbiosis. J Nutr Biochem 66:43-51.

98. Wang D, Ho L, Faith J, Ono K, Janl EM, Lachcik PJ, et al. (2015): Role of intestinal microbiota in the generation of polyphenol-derived phenolic acid mediated attenuation of Alzheimer's disease $\beta$-amyloid oligomerization. Mol Nutr Food Res 59:1025-1040. 